\title{
A Spectral Collocation Method for Acoustic Scattering by Multiple Elastic Plates
}

\author{
Matthew J Colbrook, Lorna J Ayton \\ Department of Applied Mathematics and Theoretical Physics, University of Cambridge, \\ Wilberforce Road, CB3 OWA, UK
}

\begin{abstract}
This paper presents a new approach to solving acoustic scattering problems: the Unified Transform method. This spectral, boundary-based collocation method can be readily applied to acoustic scattering by disjoint two-dimensional structures, and, for the purposes of this paper, is illustrated in the case of multiple flat plates, which also addresses the additional difficulty of mathematical singularities in the scattered field due to diffraction at sharp edges. Fluid-structure interaction may also be incorporated into the method, such as plate elasticity, which when applied to aerofoil trailing edges, is known to reduce aerodynamic noise. While a range of examples are illustrated to show the versatility of the method, attention is in particular given to the scattering of quadrupole sources by rigid plates with finite elastic extensions. It is seen that whilst a fully elastic plate is most beneficial acoustically, plates with only small extensions can considerably reduce the far-field sound power versus a fully rigid plate.
\end{abstract}

Keywords: acoustic scattering, elastic plates, spectral method

\section{Introduction}

The interaction of acoustic or hydrodynamic fluctuations with thin elastic structures arises in numerous situations, including both aeroacoustics, where elasticity of a wing is known to reduce the aerodynamic noise scattered by the sharp trailing edge, and oceanography, where ice sheets deform elastically

Email addresses: m.colbrook@damtp.cam.ac.uk (Matthew J Colbrook), 1.j.ayton@damtp.cam.ac.uk (Lorna J Ayton) 
on the ocean surface affecting acoustic scattering in the ocean beneath, or, where ocean waves interact with flexible marine platforms. Accurate and fast modelling of the fluid-structure interaction is key to predicting the effect of external forces on an elastic plate, or the effect of elasticity on the radiated field, and thus crucial for providing insight into a wide range of fluid dynamic problems.

When elastic structures can be approximated by flat plates, theoretical progress for scattering problems is possible predominantly through the use of the Wiener-Hopf method [19]. Examples of this relevant to the scattering of aerodynamic noise are given by [16], [23] and [14], and examples relevant to ocean acoustics by [27], [29], and recently [26]. In both applications, the factorisation required during the Wiener-Hopf method is difficult due to the complexity of the elastic kernel function [8]. In the case where the elastic plate is finite, the Wiener-Hopf method becomes more difficult still due to the need to factorise a matrix kernel rather than a scalar kernel, as seen in recent work [1]. There, the solution is approximated and obtained only in a restricted region of the scattered domain.

Numerical simulations are also complexified by the elastic properties; current work [3] for the scattering of a near-field source by a finite perforated elastic flat plate requires two problems to be solved; one for the structural modes of the plate which is done via a spectral method; the second for the scattering of the acoustic source which is achieved via a boundary element method (BEM). A benefit of this approach compared to theoretical modelling is that finite length effects are easily included, which is important as both backscattering [18] of the trailing-edge field by the leading edge, and structural resonances can be significant in aeroacoustic applications.

Inspired by the previous numerical method for elastic plates, [3], this paper presents an alternative spectral boundary-based method for obtaining the scattered field due to the interaction of acoustic sources with finite elastic plates, adapted from a similar approach used for rigid plates [5]. This approach, centred around taking the unified transform [11] (also known as the Fokas transform) of the governing equations both for the structural and acoustic fields, allows us to combine these equations in spectral space and avoids having to calculate singular integrals as is often problematic for BEM codes. It is also fast, with suitable basis functions leading to rapid convergence (see [5] for comparisons with other spectral and boundary based methods). Our new method can be easily extended to more complicated geometries, such as those consisting of multiple, not necessarily parallel plates, 
and therefore could provide a valuable tool for a range of aeroacoustic and hydroacoustic applications. Additionally, different sources could easily be input for this method. However, such a consideration is beyond the scope of the current paper.

The layout of this paper is as follows. Section 2 outlines the mathematical problem of the scattering of acoustic waves by finite plates with flexural elasticity. Section 3 contains details on the unified transform, both generally and specific to scattering by collinear elastic plates (which is the focus of this paper and provides some simplification to the general method). We also discuss the choice of basis functions in which we expand the unknown boundary data, and collocation points most amenable for fast computation of the resulting linear system when boundaries deform due to wave-bearing elasticity. We further provide a numerical analysis of our new approach and discuss its advantages over traditional methods. Section 4 illustrates the utility of the new method with a range of examples. First, we consider the scattering of a near-field quadrupole source by a finite elastic plate (achieved through the acoustic reciprocity principle) and compare to previous results [3]. Second, we illustrate the versatility of the method by including multiple elastic plates as could be useful for modelling formation flight/swimming, or multiple ice sheets. Finally, we consider joined plates, in particular, a plate consisting of a rigid section and an elastic section (clamped to the rigid section). This has particular relevance to the practical modelling of reduced trailing-edge noise designs. Our conclusions are given in Section 5.

\section{Mathematical Modelling}

We consider the scattering of some incident pressure field, $p_{I}^{*}$, by a number of finite elastic plates in an unbounded quiescent fluid of density $\rho_{0}^{*}$ and speed of sound $c_{0}^{*}$, where the superscript ${ }^{*}$ denotes a dimensional quantity. We suppose the $i$ th elastic plate is parallel to the horizontal $x^{*}$ direction and is described by a contour $\gamma_{i}$. We non-dimensionalise lengths by the length of a typical plate, velocities by $c_{0}^{*}$, and pressures by $\rho_{0}^{*} c_{0}^{* 2}$, denoting non-dimensional quantities without the ${ }^{*}$ superscript.

We suppose the incident pressure field is given by $p_{I}(x, y, t)=q_{I}(x, y) \mathrm{e}^{-\mathrm{i} \omega t}$ and the scattered pressure field is denoted by $p(x, y, t)=q(x, y) \mathrm{e}^{-\mathrm{i} \omega t}$. The scattered field thus satisfies

$$
\frac{\partial^{2} q}{\partial x^{2}}+\frac{\partial^{2} q}{\partial y^{2}}+k_{0}^{2} q=0
$$


subject to the Sommerfeld radiation condition, and appropriate boundary conditions on each elastic plate. By denoting the deformation of the $i$ th elastic plate by $\eta_{i}(x) \mathrm{e}^{-\mathrm{i} \omega t}$, the dynamic condition on each plate satisfies

$$
\left(\frac{\partial^{4}}{\partial x^{4}}-\frac{k_{0}^{4}}{\Omega_{i}^{4}}\right) \eta_{i}=-\frac{\epsilon_{i}}{\Omega_{i}^{6}} k_{0}^{3}[q] \quad \text { on } \gamma_{i},
$$

where $\epsilon_{i}$ is the fluid loading parameter, given by $\epsilon=\rho_{0} k_{0} /\left(m k_{B}\right)^{2}$ and $\Omega_{i}=$ $k_{0} / k_{B}$ the vacuum bending wave Mach number for the $i$ th plate [3]. Here, $m$ is the specific mass of the plate, and $k_{B}$ is the in-vacuo bending wavenumber of the plate. Finally, $[q]$ denotes the jump in pressure across the plate (pressure above the plate minus pressure below the plate).

The kinematic condition for each plate is given by

$$
k_{0}^{2} \eta_{i}=\frac{\partial q_{I}}{\partial y}+\frac{\partial q}{\partial y} \quad \text { on } \quad \gamma_{i}
$$

The ends of each plate will be specified as either clamped or free. Supposing the end of an elastic plate is at $x=x_{0}$, it is clamped if

$$
\eta\left(x_{0}\right)=\eta^{\prime}\left(x_{0}\right)=0
$$

or it is free if

$$
\eta^{\prime \prime}\left(x_{0}\right)=\eta^{\prime \prime \prime}\left(x_{0}\right)=0 .
$$

Finally, should an additional (stationary) rigid surface be present along the contour $\Gamma$, also parallel to the $x$ axis, we require

$$
\frac{\partial q}{\partial y}=-\frac{\partial q_{I}}{\partial y} \quad \text { on } \quad \Gamma .
$$

\section{Unified Transform}

In this section, we discuss first how to generate the so-called 'global relation' obtained from the unified transform, which then permits numerical progress. Once the global relation is obtained, we can expand all unknown boundary data in terms of carefully selected basis functions to obtain a linear system for the expansion coefficients. Finally, the linear system can be evaluated at collocation points to solve for the unknown expansion coefficients.

We begin by discussing the general theory behind the unified transform in $\S 3.1$, before simplifying to the case of collinear plates in $\S 3.2$. We discuss the choice of basis functions in $\S 3.3$, and collocation points in $\S 3.4$. A simple example with three rigid plates is then given in $\S 3.5$. 


\subsection{General theory}

The unified transform can be applied to arbitrary elliptic PDEs with constant coefficients [5, 6, 7] and more general separable PDEs [4], with boundary conditions prescribed on curved boundaries, non-flat bodies and bodies close to a source $[4,9,10,17]$. However, for the sake of brevity we shall describe the method applied to the Helmholtz equation Eq. (1). First, let $v$ be another solution to the Helmholtz equation. Multiplying Eq. (1) by $v$, and then subtracting the same equation with $q$ and $v$ interchanged we find

$$
\frac{\partial}{\partial x}\left(v \frac{\partial q}{\partial x}-q \frac{\partial v}{\partial x}\right)+\frac{\partial}{\partial y}\left(v \frac{\partial q}{\partial y}-q \frac{\partial v}{\partial y}\right)=0 .
$$

If the equations are valid over a domain $\mathcal{D}$ and the contributions at infinity vanish, Green's theorem (see for example [22]) together with Eq. (7) imply that

$$
\int_{\partial \mathcal{D}}\left[\left(v \frac{\partial q}{\partial x}-q \frac{\partial v}{\partial x}\right) \mathrm{d} y-\left(v \frac{\partial q}{\partial y}-q \frac{\partial v}{\partial y}\right) \mathrm{d} x\right]=0 .
$$

In order to express the integrand of the above equation in terms of just the Dirichlet and Neumann boundary values, we parametrize $q(x, y)$ and $v(x, y)$ in terms of the arc length, $s$, of $\partial \mathcal{D}$. Differentiating the function $q(x(s), y(s))$ with respect to $s$ we find

$$
\frac{\partial q}{\partial x} \mathrm{~d} y-\frac{\partial q}{\partial y} \mathrm{~d} x=q_{n} \mathrm{~d} s
$$

where $q_{n}$ denotes the derivative of $q$ along the outward normal to the boundary. Inserting Eq. (9) into Eq. (8), we find the general global relation

$$
\int_{\partial \mathcal{D}}\left(v \frac{\partial q}{\partial n}-q \frac{\partial v}{\partial n}\right) \mathrm{d} s=0
$$

In what follows, in order to further simplify the global relation, we introduce the complex variable $z=x+\mathrm{i} y$, and its conjugate $\bar{z}=x-\mathrm{i} y$. This enables us to write the Helmholtz equation in the form

$$
\frac{\partial^{2} q}{\partial z \partial \bar{z}}+\beta^{2} q=0
$$

where $\beta=k_{0} / 2$. We make the particular choice $v=\mathrm{e}^{-\mathrm{i} \beta\left(\lambda z+\frac{\bar{z}}{\lambda}\right)}$, a convenient and simple parametrisation of solutions to the Helmholtz equation obtained 


$\frac{\gamma_{1}}{x_{1,0}} x_{1,1} \frac{\gamma_{2}}{x_{2,0}} x_{2,1} \quad \frac{\gamma_{3}}{x_{3,0}} \quad x_{3,1} \quad y=0$

Figure 1: Example of the geometry and labelling convention for three collinear plates.

using separation of variables (and consistent with the literature on the unified transform). Then, Eq. (10) gives the global relation

$$
\int_{\partial \mathcal{D}} \mathrm{e}^{-\mathrm{i} \beta\left(\lambda z+\frac{\bar{z}}{\lambda}\right)}\left[q_{n}+\beta\left(\lambda \frac{\mathrm{d} z}{\mathrm{~d} s}-\frac{1}{\lambda} \frac{\mathrm{d} \bar{z}}{\mathrm{~d} s}\right) q\right] \mathrm{d} s=0 .
$$

Note that this relation involves only $q$ and its normal derivative, $q_{n}$, on the boundary. The range of validity of the complex parameter $\lambda$ depends on the domain $\mathcal{D}$. If the domain is bounded, the global relation is valid for all complex $\lambda$ (excluding 0 ), since each integral along the boundary always converges. If the domain is unbounded, then the range of values of $\lambda$ for which the integrals converge and contributions at infinity vanish are limited to ensure the validity of Green's theorem. For example, $\operatorname{Re}\left[-\mathrm{i} \beta\left(\lambda z+\frac{\bar{z}}{\lambda}\right)\right]<0$. Other values can also be included by assuming $q$ satisfies the Sommerfeld radiation condition, since the convergence of the integral is then provided by the solution, $q$ itself (see [25] for further details).

In this paper we are primarily concerned with the scattering of acoustic sources by a finite collection of collinear plates $\gamma=\cup_{i=1}^{M} \gamma_{i}$, where each plate is located at $y=0$ and $x \in\left[x_{i, 0}, x_{i, 1}\right]=\gamma_{i}$ with $x_{i, 0}<x_{i, 1} \leq x_{i+1,0}$. Thus our total scattering domain consists of $\mathbb{R}^{2} \backslash \gamma$. An example of the set up for three plates is shown in Figure 1. We discuss this case in the following subsection.

\subsection{The global relation for collinear plates}

In order to employ the unified transform on $\mathcal{D}=\mathbb{R}^{2} \backslash \gamma$ we consider two separate semi-infinite domains:

$$
\begin{aligned}
& \mathcal{D}_{1}=\left\{-\infty<x<\infty, \quad 0_{+} \leq y<\infty\right\}, \\
& \mathcal{D}_{2}=\left\{-\infty<x<\infty, \quad-\infty<y \leq 0_{-}\right\}
\end{aligned}
$$

We apply the global relation, Eq. (12), separately to $\mathcal{D}_{1,2}$.

Evaluating the global relation Eq. (12) in the domains $\mathcal{D}_{1}$ and $\mathcal{D}_{2}$, yields the following two relations. Namely, from integrating along $\partial \mathcal{D}_{1}$ we obtain 
the relation

$$
\begin{aligned}
& \int_{\mathbb{R} \backslash \gamma} \mathrm{e}^{-\mathrm{i} \beta x\left(\lambda+\frac{1}{\lambda}\right)}\left[-q_{y}\left(x, 0_{+}\right)+\beta\left(\lambda-\frac{1}{\lambda}\right) q\left(x, 0_{+}\right)\right] \mathrm{d} x \\
& +\int_{\gamma} \mathrm{e}^{-\mathrm{i} \beta x\left(\lambda+\frac{1}{\lambda}\right)}\left[-q_{y}\left(x, 0_{+}\right)+\beta\left(\lambda-\frac{1}{\lambda}\right) q\left(x, 0_{+}\right)\right] \mathrm{d} x=0,
\end{aligned}
$$

valid for $\lambda \in(-\infty,-1) \cup(0,1) \cup\left\{\mathrm{e}^{\mathrm{i} \theta}: 0<\theta<\pi\right\}=\Lambda_{1}$. Similarly, from integrating along $\partial \mathcal{D}_{2}$ we obtain the relation

$$
\begin{aligned}
& \int_{\mathbb{R} \backslash \gamma} \mathrm{e}^{-\mathrm{i} \beta x\left(\lambda+\frac{1}{\lambda}\right)}\left[q_{y}\left(x, 0_{-}\right)-\beta\left(\lambda-\frac{1}{\lambda}\right) q\left(x, 0_{-}\right)\right] \mathrm{d} x \\
& +\int_{\gamma} \mathrm{e}^{-\mathrm{i} \beta x\left(\lambda+\frac{1}{\lambda}\right)}\left[q_{y}\left(x, 0_{-}\right)-\beta\left(\lambda-\frac{1}{\lambda}\right) q\left(x, 0_{-}\right)\right] \mathrm{d} x=0,
\end{aligned}
$$

valid for $\lambda \in(-1,0) \cup(1, \infty) \cup\left\{\mathrm{e}^{\mathrm{i} \theta}: \pi<\theta<2 \pi\right\}=\Lambda_{2}$.

With these two equations, Eq. (13) and Eq. (14), we cannot impose all of our boundary conditions since the boundary conditions rely on the jump of $q$ across $y=0$. We must therefore combine Eq. (13) and Eq. (14) in order to apply the appropriate boundary conditions given in $\S 2$. We cannot currently combine Eq. (13) and Eq. (14) as they are valid in different regions of the complex $\lambda$-plane, thus we must define a symmetry transform, $\lambda \rightarrow \lambda^{-1}$ which $\operatorname{maps} \Lambda_{1} \leftrightarrow \Lambda_{2}$.

We manipulate Eq. (13) and Eq. (14) using the symmetry transform. Namely, we subtract the symmetry transform of Eq. (13) from Eq. (14) to obtain

$$
\begin{aligned}
& \int_{\mathbb{R} \backslash \gamma} \mathrm{e}^{-\mathrm{i} \beta x\left(\lambda+\frac{1}{\lambda}\right)} q_{y}(x, 0) \mathrm{d} x \\
& +\int_{\gamma} \mathrm{e}^{-\mathrm{i} \beta x\left(\lambda+\frac{1}{\lambda}\right)}\left[q_{y}(x, 0)+\frac{\beta}{2}\left(\lambda-\frac{1}{\lambda}\right)[q](x, 0)\right] \mathrm{d} x=0, \quad \lambda \in \Lambda_{2},
\end{aligned}
$$

where we have also used the fact that $q_{y}$ is continuous across the plates (i.e. the fluid is in constant contact with the oscillating plate) and continuity of $q, q_{y}$ across $\mathbb{R} \backslash \gamma$ (continuity of pressure and vertical velocity when no plates are present). Thus, Eq. (15) gives us the most simplified form of the global relation suitable for solving the Helmholtz equation with boundary conditions prescribed on collinear sections, $\gamma_{i}$.

The idea now is to expand the unknowns in this relation in suitable basis functions and evaluate at enough collocations points $\lambda$ to set up a linear system for the unknown coefficients. This is explained in the next subsection. 


\subsection{Basis functions and the approximate global relation}

For convenience, we split $\mathbb{R} \backslash \gamma$ into the following intervals. Let $I_{1}=$ $\left(-\infty, x_{1,0}\right)$ and $I_{2}=\left(x_{M, 1}, \infty\right)$. Then $\left[x_{1,0}, x_{M, 1}\right] \backslash \gamma$ can be split into disjoint open intervals of the form $J_{i}=\left(x_{i, 2}, x_{i, 3}\right)$ for $i=1, \ldots, M^{\prime}$ where $M^{\prime}$ is at most $M-1$. Note that if two plates touch $\left(x_{i, 1}=x_{i+1,0}\right.$ for some $\left.i\right)$ then we have $M^{\prime}<M-1$. For notational convenience, we introduce $L_{i}=\left|\gamma_{i}\right|=x_{i, 1}-x_{i, 0}$ and $\mu_{i}=\left(x_{i, 1}+x_{i, 0}\right) / 2$ for $i=1, \ldots, M$ as well as $L_{i}^{\prime}=\left|J_{i}\right|$ and $\mu_{i}^{\prime}$ equal to the midpoint of $J_{i}$ for $i=1, \ldots, M^{\prime}$.

\subsubsection{Rigid plates}

To illustrate our method, consider first the case of $M$ disjoint rigid plates. In this case, the unknowns in the global relation Eq. (15) are $[q]$ on each interval $\gamma_{i}$ and $q_{y}$ on $I_{1}, I_{2}$ and on each $J_{i}$. For the finite intervals $\gamma_{i}$, in order to capture the $\sqrt{1-t^{2}}$ type singularity near the edge tips we define

$$
C_{m}(t)=\sqrt{1-t^{2}} U_{m}(t),
$$

where $U_{m}(\cdot)$ denote Chebyshev polynomials of the second kind. These have the following Fourier transform:

$$
\int_{-1}^{1} \mathrm{e}^{\mathrm{i} \lambda t} \sqrt{1-t^{2}} U_{m}(t) \mathrm{d} t=\frac{(m+1) \mathrm{i}^{m} \pi}{\lambda} J_{m+1}(\lambda),
$$

where $J_{\alpha}(\cdot)$ denotes the Bessel function of the first kind of order $\alpha$. We expand $\left[q_{i}\right]=[q]$ on $\gamma_{i}$ as

$$
\left[q_{i}\right](x) \approx \sum_{n=1}^{N_{i}} a_{i, n} C_{n-1}\left(\frac{2 x-2 \mu_{i}}{L_{i}}\right) .
$$

Similarly, for the $M^{\prime}$ intervals $J_{i}$ we expand $q_{y, i}=q_{y}$ as

$$
q_{y, i}(x) \approx \sum_{n=1}^{N_{i}^{\prime}} b_{i, n} S_{n-1}\left(\frac{2 x-2 \mu_{i}^{\prime}}{L_{i}^{\prime}}\right)
$$

where

$$
S_{m}(t)=\frac{T_{m}(t)}{\sqrt{1-t^{2}}}
$$


and $T_{m}(\cdot)$ denote Chebyshev polynomials of the first kind. These are chosen to capture the derivatives of the relevant square root type singularity and have

$$
\int_{-1}^{1} \mathrm{e}^{\mathrm{i} \lambda t} \frac{T_{m}(t)}{\sqrt{1-t^{2}}} \mathrm{~d} t=\mathrm{i}^{m} \pi J_{m}(\lambda)
$$

For the semi-infinite intervals $I_{1}$ and $I_{2}$ we expand (after a relevant linear change of variables) in terms of the Bessel functions $\left\{J_{\frac{n+1}{2}}\left(k_{0} x\right) / x\right\}_{n \geq 0}$. These have the advantage of capturing the correct singular behaviour near the plate edges when $n$ is even. They decay with the correct algebraic rate at infinity, and have easy to compute Fourier transforms [20]:

$$
\int_{0}^{\infty} \mathrm{e}^{\mathrm{i} \lambda t} \frac{J_{\alpha}(b t)}{t} \mathrm{~d} t=\left\{\begin{array}{ll}
\frac{\exp (\mathrm{i} \alpha \arcsin (\lambda / b))}{\alpha}, & \text { for } 0 \leq \lambda \leq b \\
\frac{b^{\alpha} \exp (\alpha \pi \mathrm{i} / 2)}{\alpha\left(\lambda+\sqrt{\lambda^{2}-b^{2}}\right)^{\alpha}}, & \text { for } 0<b \leq \lambda
\end{array} .\right.
$$

Explicitly, we approximate $q_{y, 0}=q_{y}$ on $I_{1}$ via

$$
q_{y, 0} \approx \sum_{n=1}^{N_{0}^{\prime}} b_{0, n} \frac{J_{\frac{n}{2}}\left(k_{0}\left(x_{1,0}-x\right)\right)}{x_{1,0}-x}
$$

and $q_{y, M^{\prime}+1}=q_{y}$ on $I_{2}$ via

$$
q_{y, 0} \approx \sum_{n=1}^{N_{M^{\prime}+1}^{\prime}} b_{M^{\prime}+1, n} \frac{J_{\frac{n}{2}}\left(k_{0}\left(x-x_{M, 1}\right)\right)}{x-x_{M, 1}}
$$

Using the formulae for the relevant Fourier transforms, we thus form an approximate global relation

$$
\sum_{i=1}^{M} \sum_{n=1}^{N_{i}} A_{i, n}(\lambda) a_{i, n}+\sum_{i=0}^{M^{\prime}+1} \sum_{n=1}^{N_{i}^{\prime}} B_{i, n}(\lambda) b_{i, n} \approx \int_{\gamma} \mathrm{e}^{-\mathrm{i} \beta x\left(\lambda+\frac{1}{\lambda}\right)} \frac{\partial q_{I}}{\partial y}(x, 0) \mathrm{d} x .
$$

The coefficients $A$ are given by

$$
A_{i, n}(\lambda)=\left(\frac{1}{\lambda}-\lambda\right) \mathrm{e}^{-\mathrm{i} \beta \mu_{i}(\lambda+1 / \lambda)} \frac{n \mathrm{i}^{n-1} \pi}{2\left(\lambda+\frac{1}{\lambda}\right)} J_{n}\left(-\frac{\beta L_{i}}{2}\left(\lambda+\frac{1}{\lambda}\right)\right) .
$$


We also have

$$
\begin{aligned}
B_{0, n}(\lambda) & =\mathrm{e}^{-\mathrm{i} \beta x_{1,0}(\lambda+1 / \lambda)} \int_{0}^{\infty} \mathrm{e}^{\mathrm{i} \beta t(\lambda+1 / \lambda)} \frac{J_{\frac{n}{2}}\left(k_{0} t\right)}{t} \mathrm{~d} t, \\
B_{M^{\prime}+1, n}(\lambda) & =\mathrm{e}^{-\mathrm{i} \beta x_{M, 1}(\lambda+1 / \lambda)} \int_{0}^{\infty} \mathrm{e}^{-\mathrm{i} \beta t(\lambda+1 / \lambda)} \frac{J_{\frac{n}{2}}\left(k_{0} t\right)}{t} \mathrm{~d} t .
\end{aligned}
$$

Finally, for $0<i<M^{\prime}$ we have

$$
B_{i, n}=\frac{L_{i}^{\prime} i^{n-1} \pi}{2} \mathrm{e}^{-\mathrm{i} \beta \mu_{i}^{\prime}(\lambda+1 / \lambda)} J_{n-1}\left(-\frac{\beta L_{i}^{\prime}}{2}\left(\lambda+\frac{1}{\lambda}\right)\right)
$$

We evaluate Eq. (20) at $C \geq \sum N_{i}+\sum N_{i}^{\prime}$ points $\lambda \in \Lambda_{2}$ to set up a linear system for the unknown coefficients. This is then inverted in the least squares sense. Once the coefficients are computed, we can reconstruct approximations of the unknown functions.

\subsubsection{Elastic plates}

When dealing with elastic plates, it is advantageous to choose basis functions that obey the clamped/free boundary conditions. To explain the method, we will consider the case of a single elastic plate with $x_{1,0}=-1$ and $x_{1,1}=1$, clamped at $x=-1$ and free at $x=1$. We expand the plate deformation as

$$
\eta_{1}(x) \approx \sum_{j=1}^{N} a_{j} f_{j}(x),
$$

where the $\left\{f_{j}\right\}_{j=1}^{\infty}$ are the eigenfunctions (ordered in ascending eigenvalues) of $\nabla^{4}$ on the interval $[-1,1]$ with the corresponding boundary conditions. Namely,

$$
\nabla^{4} f_{j}=d_{j}^{4} f_{j}, \quad f_{j}(0)=f_{j}^{\prime}(0)=0, \quad f_{j}^{\prime \prime}(1)=f_{j}^{\prime \prime \prime}(1)=0,
$$

and $d_{j} \geq 0$. Computing the $\left\{f_{j}, d_{j}\right\}$ can be done using standard spectral methods $[28,2]$. We expand each $f_{j}$ in Chebyshev polynomials (we used the first 401 Chebyshev polynomials) and compute the coefficients in the Chebyshev expansion and eigenvalues using extended precision. This then gives the eigenvalues, end point values (see below) and Chebyshev coefficients of the $f_{j}$ accurate to standard machine precision. This only needs to be done 
once offline. ${ }^{1}$ Along the interval $\gamma_{1}$ we therefore have that

$$
\begin{aligned}
\frac{\partial q}{\partial y}(x) & \approx-\frac{\partial q_{I}}{\partial y}(x)+\sum_{j=1}^{N} k_{0}^{2} a_{j} f_{j}(x) \\
{[q](x) } & =-\frac{\Omega_{1}^{6}}{k_{0}^{3} \epsilon_{1}}\left(\frac{\partial^{4}}{\partial x^{4}}-\frac{k_{0}^{4}}{\Omega_{1}^{4}}\right) \eta_{1}(x) \\
& \approx-\frac{\Omega_{1}^{6}}{k_{0}^{3} \epsilon_{1}} \sum_{j=1}^{N} a_{j}\left(d_{j}^{4}-\frac{k_{0}^{4}}{\Omega_{1}^{4}}\right) f_{j}(x) .
\end{aligned}
$$

There are two advantages of using this basis. First, the boundary conditions are naturally captured and we also gain the plate displacement $\eta_{1}$ at the same time without any additional computation. Second, the Fourier transform (needed to set up the approximate global relation) is given explicitly after repeated integration by parts as

$$
\begin{aligned}
\left(\lambda^{4}-d_{j}^{4}\right) \int_{-1}^{1} \mathrm{e}^{\mathrm{i} \lambda x} f_{j}(x) \mathrm{d} x & =(\mathrm{i} \lambda)^{3}\left[\mathrm{e}^{\mathrm{i} \lambda x} f(x)\right]_{x=-1}^{1}-(\mathrm{i} \lambda)^{2}\left[\mathrm{e}^{\mathrm{i} \lambda x} f^{\prime}(x)\right]_{x=-1}^{1} \\
& +\mathrm{i} \lambda\left[\mathrm{e}^{\mathrm{i} \lambda x} f^{\prime \prime}(x)\right]_{x=-1}^{1}-\left[\mathrm{e}^{\mathrm{i} \lambda x} f^{\prime \prime \prime}(x)\right]_{x=-1}^{1},
\end{aligned}
$$

where the end point values are computed from the Chebyshev expansions of the function $f_{j}$. The approximate global relation then becomes

$$
\begin{aligned}
& \sum_{j=1}^{N} a_{j} \int_{-1}^{1} \mathrm{e}^{-\mathrm{i} \beta x\left(\lambda+\frac{1}{\lambda}\right)}\left[k_{0}^{2}-\frac{\beta \Omega_{1}^{6}}{2 k_{0}^{3} \epsilon_{1}}\left(d_{j}^{4}-\frac{k_{0}^{4}}{\Omega_{1}^{4}}\right)\left(\lambda-\frac{1}{\lambda}\right)\right] f_{j}(x) \mathrm{d} x \\
& +\sum_{i=0}^{1} \sum_{n=1}^{N_{i}^{\prime}} B_{i, n}(\lambda) b_{i, n} \approx \int_{\gamma_{1}} \mathrm{e}^{-\mathrm{i} \beta x\left(\lambda+\frac{1}{\lambda}\right)} \frac{\partial q_{I}}{\partial y}(x, 0) \mathrm{d} x .
\end{aligned}
$$

The case of $\gamma_{1} \neq[-1,1]$ is dealt with via an appropriate linear change of variables (note that we can still use the eigenfunctions computed on $[-1,1]$ after an appropriate rescaling - we do not need to recompute eigenfunctions via the the spectral method for intervals of different sizes/positions). Different clamped/free boundary conditions can be treated by expanding in terms of the appropriate eigenfunctions which obey the relevant boundary conditions.

\footnotetext{
${ }^{1}$ In fact, even with the use of extended precision, this can be done very quickly.
} 
Finally, when there is more than one elastic plate or a mixture of rigid/elastic plates, we simply sum the relevant contributions in the approximate global relation.

\subsection{Collocation points and obtaining the scattered field}

Unfortunately, in contrast to standard spectral methods [2], there is no current theory describing the best choices for collocation points. ${ }^{2}$ For the problems in this paper, we have found that a good choice is to take $C_{1}$ points $\mathcal{P}=\left\{-1 /\left(C_{1}+1\right)^{6},-2^{6} /\left(C_{1}+1\right)^{6}, \ldots,-C_{1}^{6} /\left(C_{1}+1\right)^{6}\right\}$. Additionally, we took the reciprocals of the negative of these values. Finally, we also took $C_{1}$ points in $\left\{\mathrm{e}^{\mathrm{i} \theta}: 0<\theta<\pi\right\}$ with $\theta$ corresponding to equally spaced points in $(0, \pi)$, leading to $C=3 C_{1}$ collocation points overall. The complex collocation points along the unit circle are allowed precisely because the solution satisfies the Sommerfeld radiation condition so that the contribution of Green's identity along the relevant semi-circular arc vanishes in the infinite radius limit (see [25]). We found that to obtain accurate numerical solutions, we needed to sample these points, and hence, we considered the full complex solution. This corresponds to implementing the boundary conditions that make the boundary value problem well-posed.

In all of the examples encountered in this paper, the scattered field is an odd function in the $y$ variable. Hence, by considering the reflected Green's function

$G_{R}\left(x, y, x^{\prime}, y^{\prime}\right)=\frac{1}{4 \mathrm{i}}\left(H_{0}^{(1)}\left(k_{0} \sqrt{\left(x-x^{\prime}\right)^{2}+\left(y-y^{\prime}\right)^{2}}\right)-H_{0}^{(1)}\left(k_{0} \sqrt{\left(x-x^{\prime}\right)^{2}+\left(y+y^{\prime}\right)^{2}}\right)\right)$

and its normal derivative, we can write

$$
q(x, y)=\frac{\mathrm{i} k_{0} y}{4} \int_{\gamma} \frac{H_{1}^{(1)}\left(k_{0} \sqrt{\left(x-x^{\prime}\right)^{2}+y^{2}}\right)}{\sqrt{\left(x-x^{\prime}\right)^{2}+y^{2}}}[q]\left(x^{\prime}, 0\right) \mathrm{d} x^{\prime},
$$

where $H_{\alpha}^{(1)}(\cdot)$ denotes the Hankel function of the first kind of order $\alpha$. For points off the union of plates $\gamma$, we evaluated this using standard Gaussian quadrature.

\footnotetext{
${ }^{2}$ For a good choice of collocation points for bounded convex polygons, see [13], which we cannot adopt here due to the restrictions on the values of $\lambda$.
} 


\subsection{Example with 3 rigid plates}

As a simple demonstration of the method, we now briefly consider the case of three rigid plates with $\gamma_{1}=[-4,1], \gamma_{2}=[1.1,2]$ and $\gamma_{3}=[3,5]$. We chose $q_{I}=\mathrm{e}^{-i k_{0} x \cos \theta-i k_{0} y \sin \theta}$ for $k_{0}=20$ and $\theta=\pi / 4$. In this example, and throughout the paper, we set $N_{i}=N, N_{0}^{\prime}=N_{M^{\prime}+1}^{\prime}=2 N$ and all other $N_{i}^{\prime}=$ $N$. The number of collocation points is determined by $C_{1}=4\left(\sum N_{i}+\sum N_{i}^{\prime}\right)$. As a measure of error, we consider the discrete relative $L^{1}$ error computed at 201 points on each $\gamma_{i}$. Namely, given a "converged" solution $[q]$ (computed by taking larger $N$ ) and an approximation $[\tilde{q}]$, we consider

$$
E_{i}=\frac{\sum_{j=1}^{201}\left|[\tilde{q}]\left(x_{i, 0}+\frac{(j-1)\left(x_{i, 1}-x_{i, 0}\right)}{200}\right)-[q]\left(x_{i, 0}+\frac{(j-1)\left(x_{i, 1}-x_{i, 0}\right)}{200}\right)\right|}{\sum_{j=1}^{201}\left|[q]\left(x_{i, 0}+\frac{(j-1)\left(x_{i, 1}-x_{i, 0}\right)}{200}\right)\right|} .
$$

Figure 2 shows the convergence of the method with $N$, as well as the computed scattered plus incident field for $N=80$. The method is also very fast, taking a few seconds on a standard desktop computer for the computation of $[q]$ with $N=80$ (720 basis functions). We have plotted the error against $N^{2}$ with the errors shown on a logarithmic scale; the near straight lines indicate that the method converges faster than exponentially. There is also a plateau in the error before this rapid convergence kicks in. Experimentation suggests that the size of $N$ needed to reach the region of rapid convergence is proportional to $k_{0}$ (one would expect that $O\left(k_{0}\right)$ basis functions are needed to resolve the relevant oscillations of the solution). The reader is referred to figure 6 of [5] for an example of this with varying $k_{0}$. For the case of elastic plates, we cannot expect exponential convergence since the basis functions (the eigenfunctions of $\nabla^{4}$ ) are smooth and hence do not capture the appropriate endpoint singularities (at the tips of the plates). They do, however, make it much easier to incorporate the boundary conditions that couple $[q]$ to the fourth order derivative of $\eta$, hence capture the much more complicated boundary conditions when the plate is no longer stationary. Note that naively expanding the normal derivative of $q$ in suitable basis functions and then substituting this into Eq. (2) and Eq. (3) (eliminating $\eta$ ) would require the fourth order derivative of the expansion functions, making it very difficult to choose singular basis functions with such an approach. We will see that the method in the elastic case still converges algebraically, and typically yields two to three digits of accuracy for the examples considered in this paper. Such accuracy is often sufficient for the relevant applications, given 


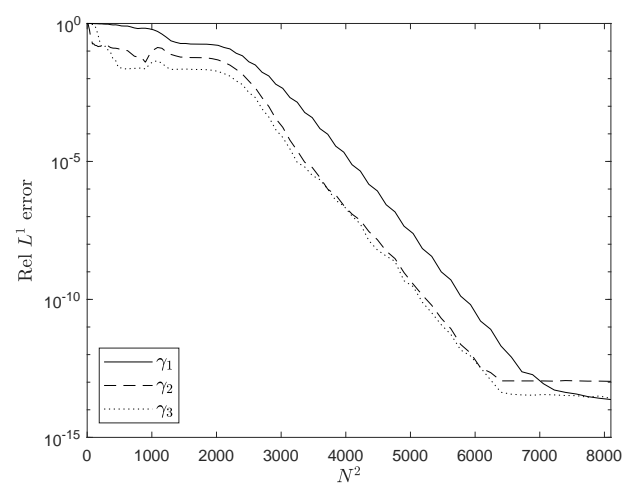

(a)
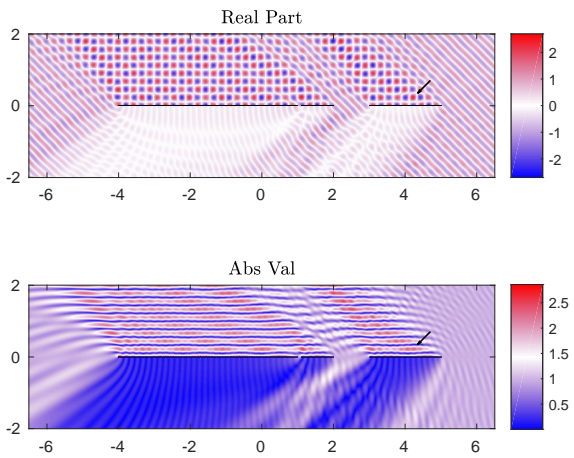

(b)

Figure 2: (a) Convergence of method for rigid plate example, errors for each plate $\gamma_{i}$ are labelled by the legend. (b) Total field computed by the method for $N=80$ at time $t=0$. The small arrow shows the direction of the incoming waves.

that the governing equations are obtained after linearisation (assuming $\eta$ to be small). Smaller errors are also possible with larger $N$.

\section{Examples and Results}

Here we present results for the acoustic scattering by a variety of elastic plate setups.

\subsection{Single elastic plate}

First, we suppose there is a single elastic plate in the region $0<x<1$, $y=0$. We suppose the end at $x=0$ is clamped, while the end at $x=1$ is free, in accordance with previous modelling [3]. We take the incident field to be a plane wave, $q_{I}=\mathrm{e}^{-i k_{0} x \cos \theta-i k_{0} y \sin \theta}$ where $\theta$ measures the polar angle counter-clockwise from the positive $x$-axis.

\subsubsection{Near-Field Quadrupole}

By invoking the acoustic reciprocity principle, evaluating $q$ close to the edge $x=1, y=0$ is equivalent to measuring the far-field acoustics at angle $\theta$ scattered by a monopole close to the edge. Similarly, evaluating $\frac{\partial q}{\partial x}$ provides the far-field pressure due to a near-field dipole, and $\frac{\partial^{2} q}{\partial x \partial y}$ the far-field pressure due to a near-field lateral quadrupole (with axes aligned with the coordinate axes). This allows us to calculate the far-field acoustic directivity, $|q|$, as a 


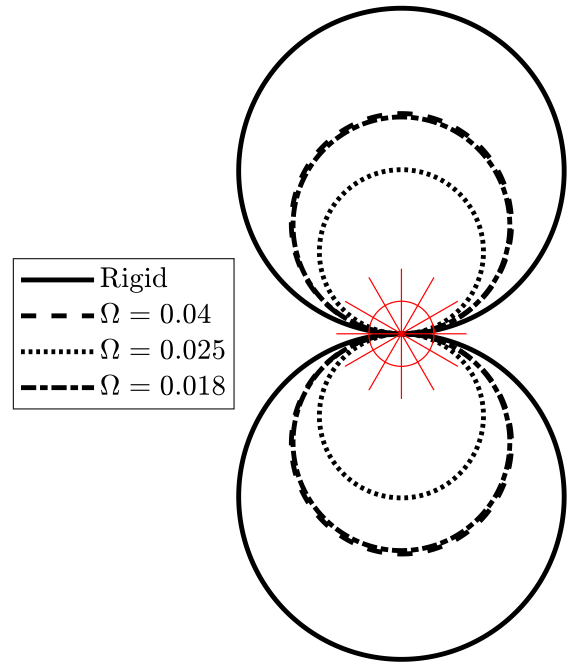

(a)

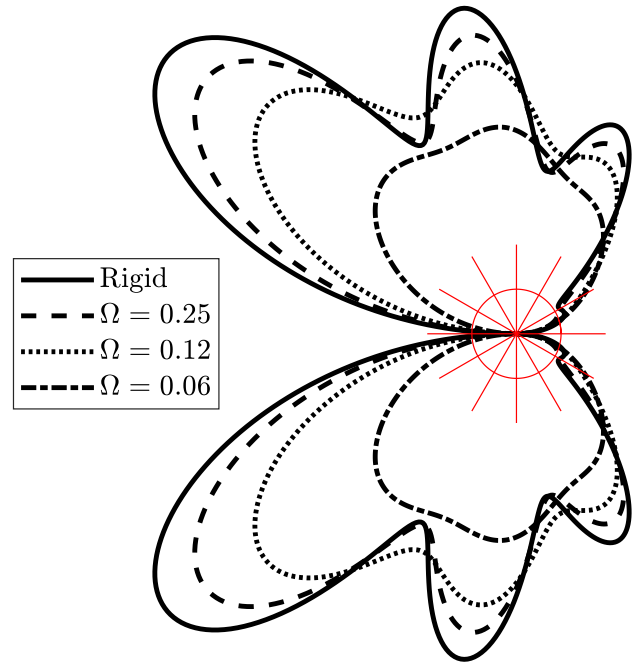

(b)

Figure 3: Far-field directivity for a lateral quadrupole located at $(1,0.005)$, for (a) $k_{0}=0.1$ and (b) $k_{0}=10$. The red lines show polar angles in increments of $30^{\circ}$. Legends denote the value of $\Omega$. The results are non-dimensional and agree well with those of [3] (figure 5).

function of observation angle, $\theta$, that would be due to a near-field trailing edge source, as would be the case for aerodynamic noise.

Figure 3 illustrates this directivity two frequencies $k_{0}=0.1,10$ in the case of $\epsilon=0.0021$ (suitable for an aluminium plate in air [15]). This compares well to previous results [3]. We see, as known from previous work, that elasticity can reduce trailing-edge noise effectively. However, the reduction in noise is not linear with increasing elasticity (decreasing $\Omega$ ) due to the elastic plate resonances. Elastic plate resonances will be discussed further in the following sections.

\subsubsection{Scattering of a plane wave}

Here we illustrate results for the full scattered field due to an incident sound wave and discuss the convergence and speed of the method. Figure 4 illustrates the total pressure field (incident plus scattered) for a plane wave scattering off a single elastic plate, while Figure 5 illustrates just the scattered field. Different plate parameters are used throughout. We see clearly in Figure 5 the decrease in the magnitude of the scattered field as the plate 


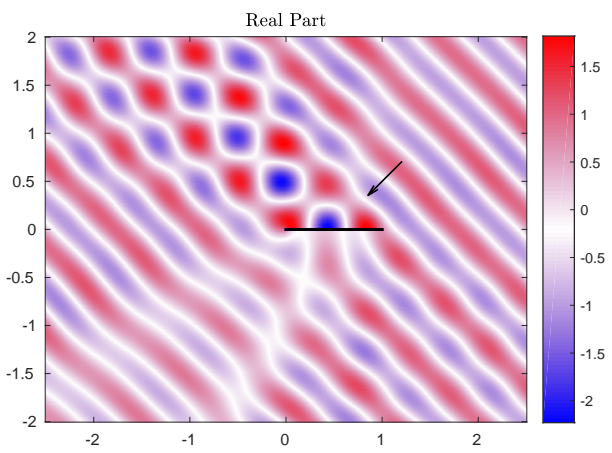

(a)

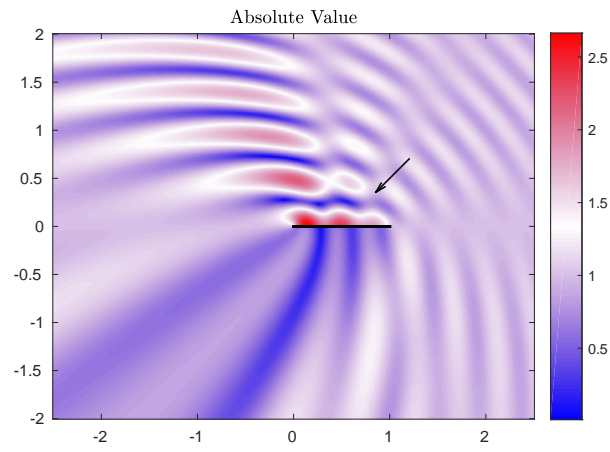

(b)

Figure 4: Total acoustic field (scattered plus incident) for $k_{0}=10, \epsilon=0.0021$ and $\Omega=0.25$. The arrow denotes the direction of propagation of the incident plane wave. We have shown (a) the real part and (b) the absolute value of the field.

becomes more flexible (more flexible corresponds to a lower value of $\Omega$ ).

From our method we can also easily recover the deformation of the plate, $\eta$, as illustrated by Figure 6 which shows the plate deformations for the acoustic fields given in Figures 4 and 5. Note the values $\epsilon=0.0021,0.135$ correspond to the fluid loading parameter for aluminium in air and water respectively [3].

Figure 7 shows the convergence of the computed values of $[q]$ and $\eta$ as we increase $N$ (computed via comparison with larger $N$ ). We note that a smaller number of modes $\eta_{j}$ are needed for smaller values of $k_{0}$. For the $k_{0}=10$ case and the computation of $\eta$, a larger number of modes are needed for smaller $\Omega$, consistent with the increase in the bending wavenumbers $k_{\mathrm{B}}=k_{0} / \Omega$. The algebraic convergence in Figure 7 is typical with similar results (and two to three digits of accuracy) for other parameter values and $N \approx 100$. Again, the method is fast, taking only a few seconds on a standard desktop computer for $N=150$ (750 basis functions).

\subsection{Multiple plates}

We now highlight the versatility of our method by introducing additional elastic plates. Whilst the focus here is on collinear elastic plates, the method can also be used for non-collinear plates (following a similar approach as for staggered rigid plates [5]) however this is beyond the scope of the current 


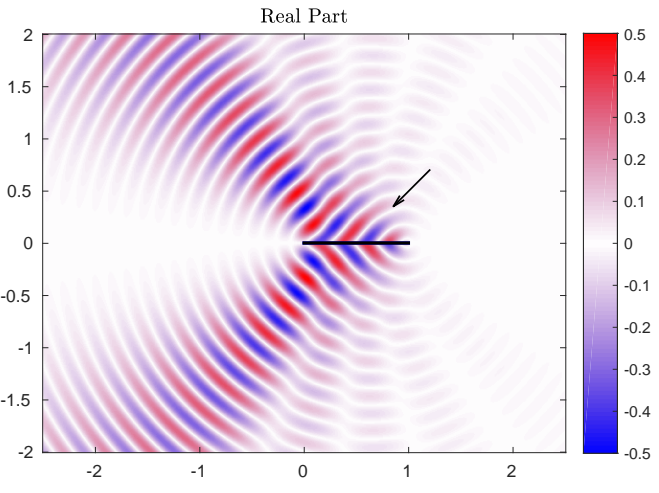

(a)

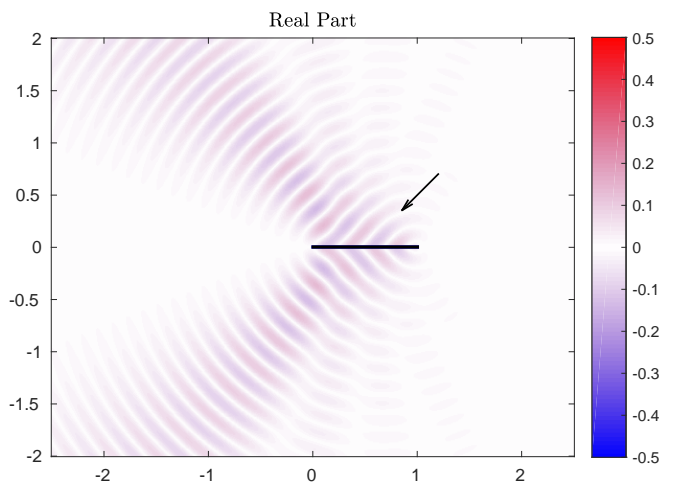

(b)

Figure 5: Scattered field for $k_{0}=20$ and $\epsilon=0.135$. The arrow denotes the direction of propagation of the incident plane wave, and the colour the real part of the scattered pressure field. The value of $\epsilon$ it typical for an aluminium plate immersed in water. The plots show (a) $\Omega=0.4$ and (b) $\Omega=0.2$.

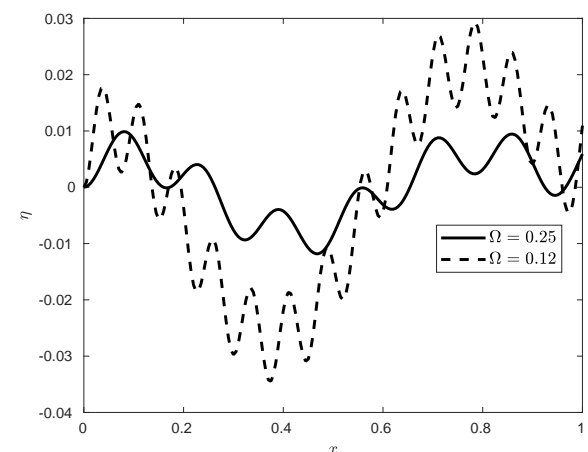

(a)

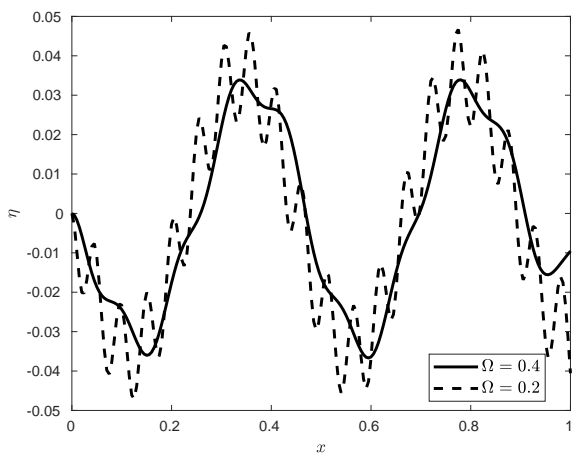

(b)

Figure 6: Deformation of the elastic plate, $\eta$, at time $t=0$. The legends denote the value of $\Omega$. The plots show (a) $\epsilon=0.0021, k_{0}=10$ and (b) $\epsilon=0.135, k_{0}=20$. 


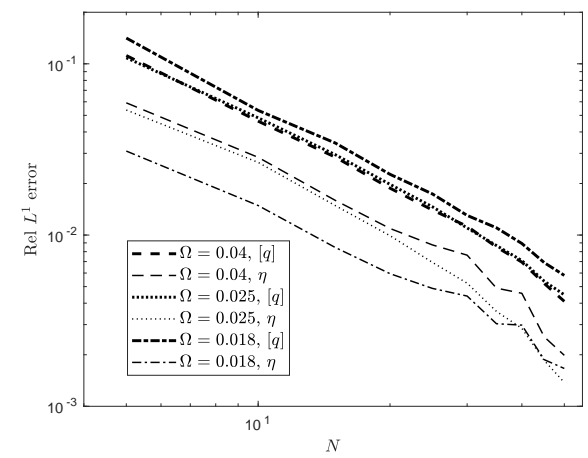

(a)

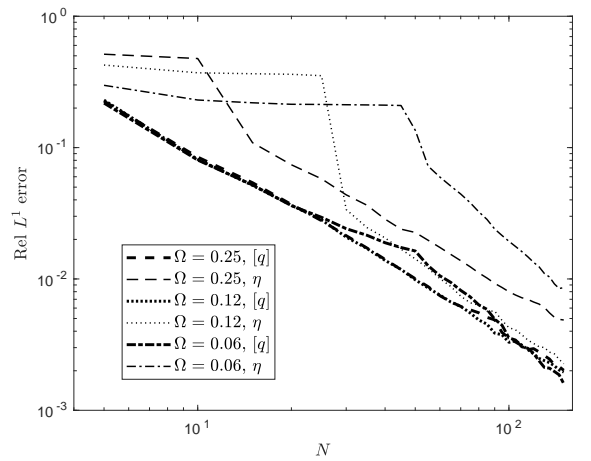

(b)

Figure 7: (a) Convergence of the computed values of $[q]$ and $\eta$ for $\epsilon=0.0021$ and $k_{0}=0.1$. (b) Same but for $\epsilon=0.0021$ and $k_{0}=10$. Both results are for $\theta=\pi / 4$.

paper. Example acoustic fields for high and low frequencies are given by Figures 8 and 9, with convergence demonstrated in Figure 10.

A large variety of multiple plate setups could be calculated with the method presented here. We shall illustrate one particular case interesting to the aeroacoustics community: elastic extensions to rigid plates. It is known that elastic plates reduce aerodynamic noise [16], however, most previous studies focus on uniformly elastic plates. Perhaps a more realistic model of the owl's wing would be a rigid section with a small elastic section at the trailing edge itself.

We therefore consider a plate of total length 1 , with an elastic section of length $l$ (so that the plate is rigid for $x \in[0,1-l]$, and elastic for $x \in[1-l, 1]$ ). The elastic plate is clamped at $x=1-l$ and free at $x=1$, and the set up is shown in Figure 11. This problem has been considered previously via the Wiener-Hopf technique, [1], although due to difficulties in factorising the matrix equations arising from the Wiener-Hopf method, results were obtained using a Padé approximation method and restricted to only the far-field directivity. Since elastic plate resonances can significantly affect the directivity when varying the plate elasticity only slightly, it is not clear from these prior results how to determine the optimal extension length and elasticity parameters for a required noise reduction.

The method presented in this paper is not only more accurate than the approximation approach [1], but can also recover the full scattered field sufficiently quickly to allow for sound power calculations. In Figure 12, we have 


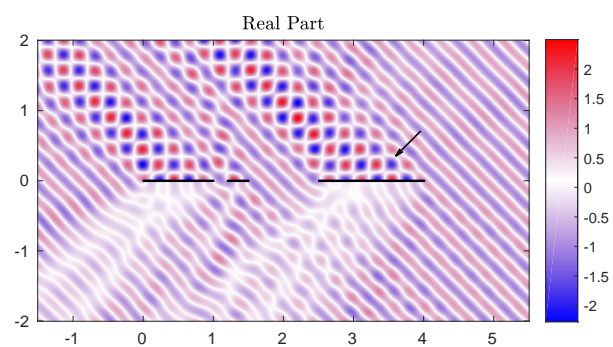

(a)

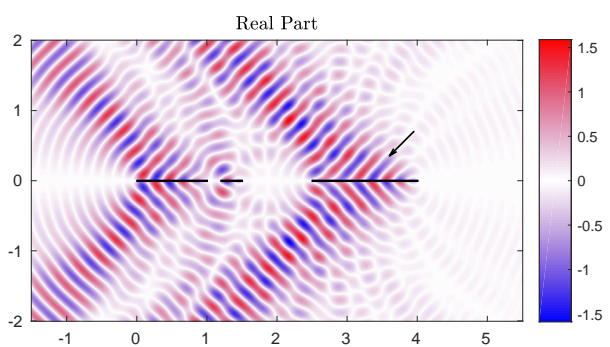

(b)

Figure 8: Acoustic fields for three plates: from left to right, elastic with $\Omega=0.4$ free at $x=0$ and clamped at $x=1$, rigid, elastic with $\Omega=0.2$, clamped at $x=2.5$ and free at $x=4$. Elastic plates both have $\epsilon=0.0021$. The incident wave has frequency $k_{0}=20$ with angle $\pi / 4$. We have shown (a) the total field and (b) the scattered field.

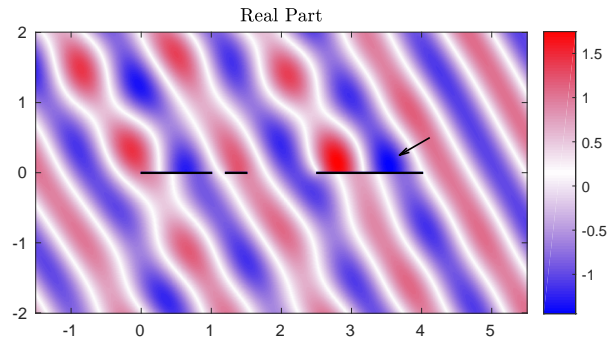

(a)

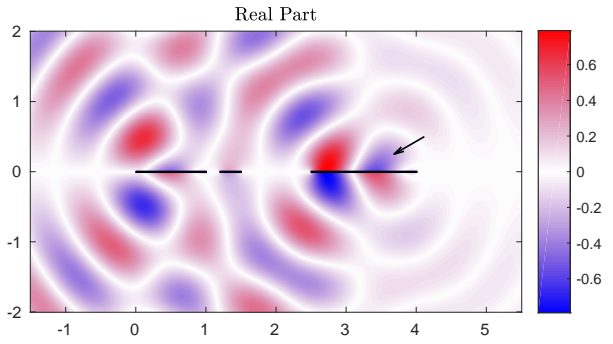

(b)

Figure 9: Acoustic fields for three plates: from left to right, elastic with $\Omega=0.2$ free at $x=0$ and clamped at $x=1$, rigid, elastic with $\Omega=0.1$, clamped at $x=2.5$ and free at $x=4$. Elastic plates both have $\epsilon=0.0021$. The incident wave has frequency $k_{0}=5$ with angle $\pi / 6$. We have shown (a) the total field and (b) the scattered field. 

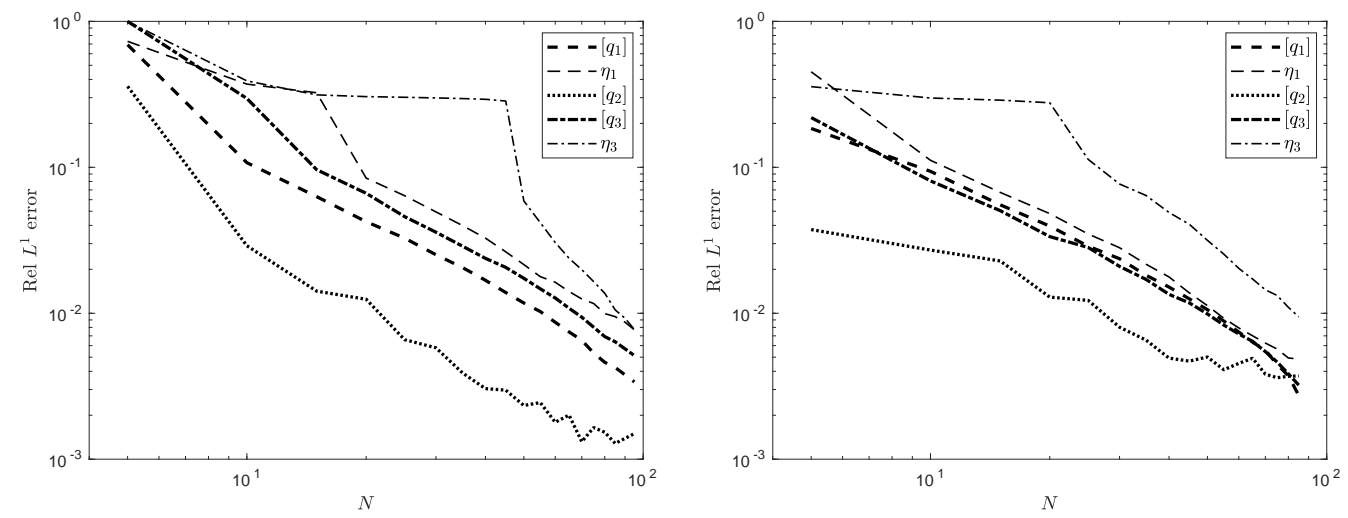

Figure 10: Convergence of the method for parameter values in (a) Figure 8 and (b) Figure 9.

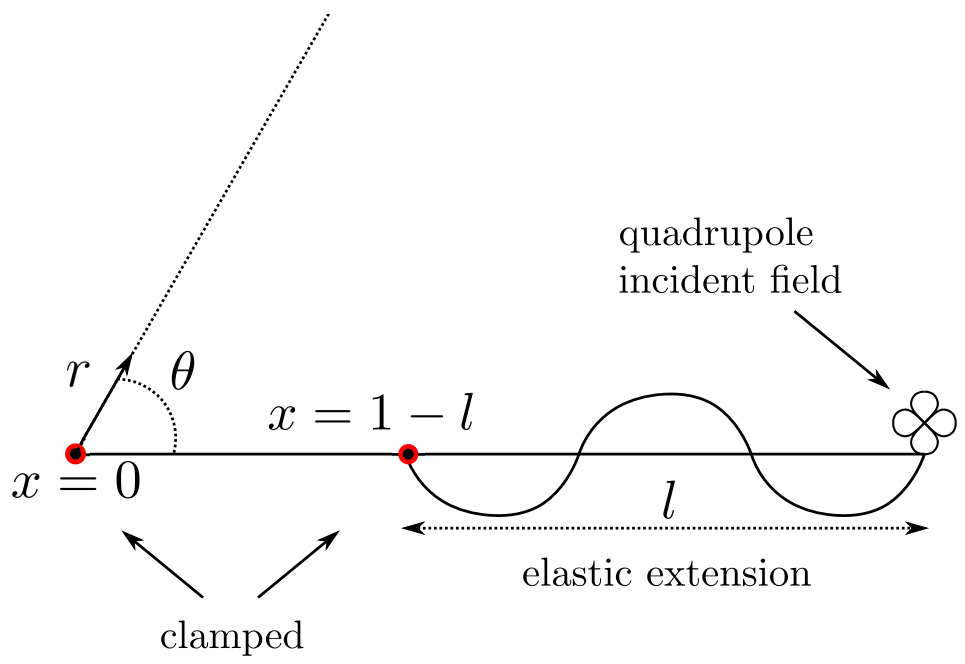

Figure 11: The problem considered for elastic extensions of rigid plates. We have shown the parameters as well as polar angle/radius. 
plotted the sound power level (normalised by dividing by the rigid plate limit) against $\Omega$ for $k_{0}=10, \epsilon=0.0021$ and different values of $l$ for a quadrupole located at $(x, y)=(1,0.02)$. We see that even for small $l$, a reduction in noise is possible. This is more striking when plotted against the dimensionless variable $l k_{\mathrm{B}}=l k_{0} / \Omega$, where we see a larger reduction in noise for smaller $l$ in terms of relative elasticity. This indicates that a short elastic section could provide ample noise reduction, rivalling the reduction of a fully elastic plate, if suitable plate parameters are chosen.

Note, that the spikes in Figure 12 correspond to the resonances of the elastic plate, and agree well with the resonance wavenumbers $d_{j}$. One could, therefore, use these plots to determine parameters for a required noise reduction and avoid plate resonances.

Finally, in Figure 13 we show the far-field directivity, again due to a quadrupole located at $x=1, y=0.02$ above a plate, for different $l$ and $\Omega$, and for $k_{0}=5,50$. We see that for the lower frequency, $k_{0}=5$, as $l$ approaches zero, the directivity is close to that of the rigid plate, even for relatively small $\Omega$ (corresponding to more flexible plates), since the acoustic field cannot excite such a short plate to oscillate. For the larger wavenumber $k_{0}=50$, even the shortest plate, $l=0.05$ can be excited if sufficiently flexible $(\Omega=0.07)$, thus a reduction in far-field directivity is observed. We see, however, that for fixed $\Omega$, at high frequency, $k_{0}=50$, there can be an increase in noise as $l$ decreases. This is due to the quadrupole field becoming

sufficiently close to the rigid section of the plate for the scattering from the elastic-rigid junction to be significant (of similar magnitude to the scattering by the trailing edge itself). Thus too short of a plate will have little effect on noise reduction even if it can be excited by a high-frequency field. For lower frequencies, the impact of the elastic-rigid junction is less significant due to acoustic compactness. Thus the quadrupole source primarily interacts only with the trailing edge, $x=1$, and provided the elastic plate can be excited, a noise reduction is expected.

\section{Conclusions}

In this paper, we have presented a spectral collocation method for acoustic scattering by multiple elastic plates. An expansion of the plate deformations in the vibrational modes of each plate allows the Fourier transform of the Dirichlet and Neumann boundary values to be computed and coupled in an appropriate manner. This is then used in the global relation, which sets up 


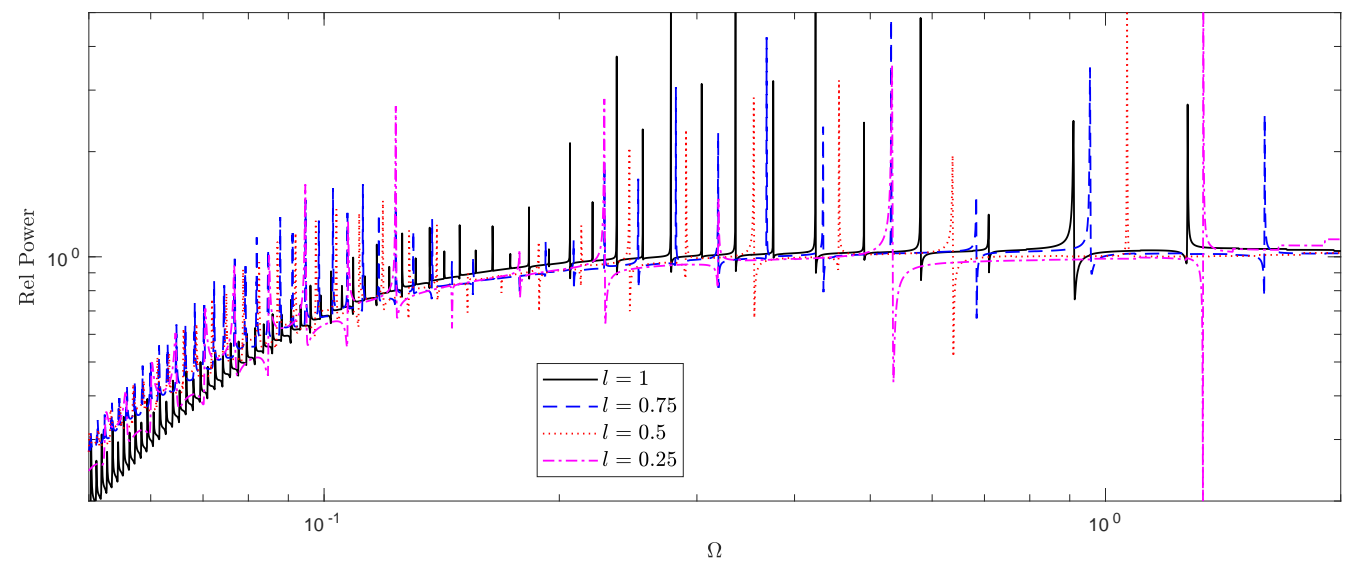

(a)

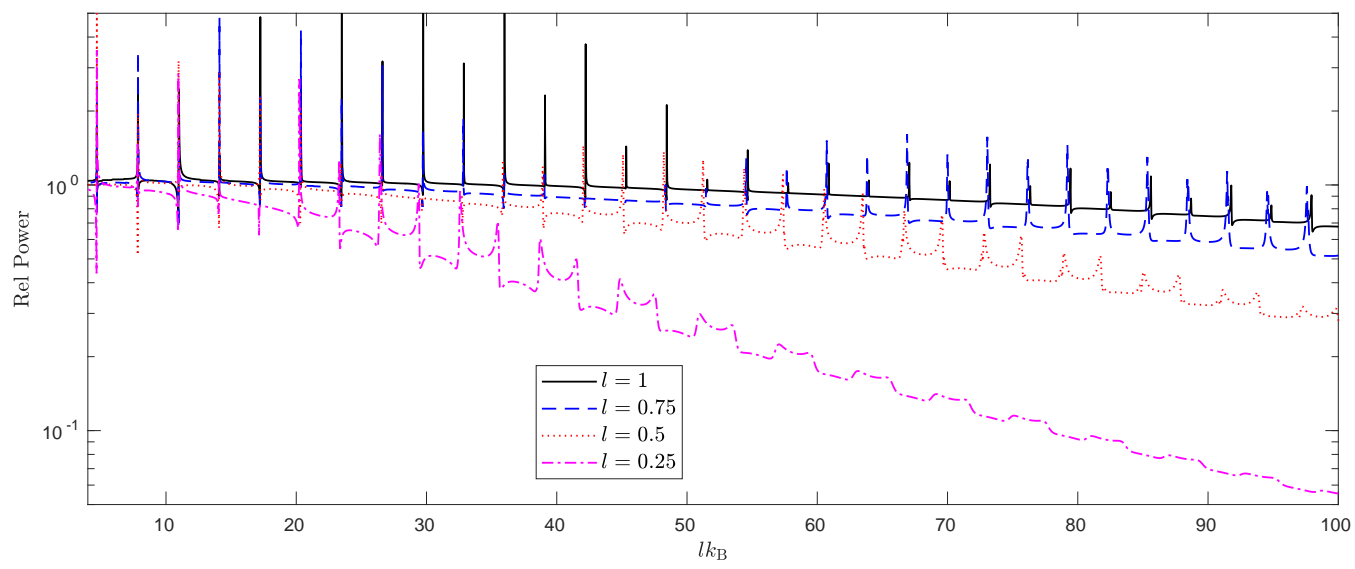

(b)

Figure 12: (a) Relative power level as a function of $\Omega$. (b) Relative power level as a function of $l k_{\mathrm{B}}$. In both cases, $k_{0}=10$ and $\epsilon=0.0021$. 

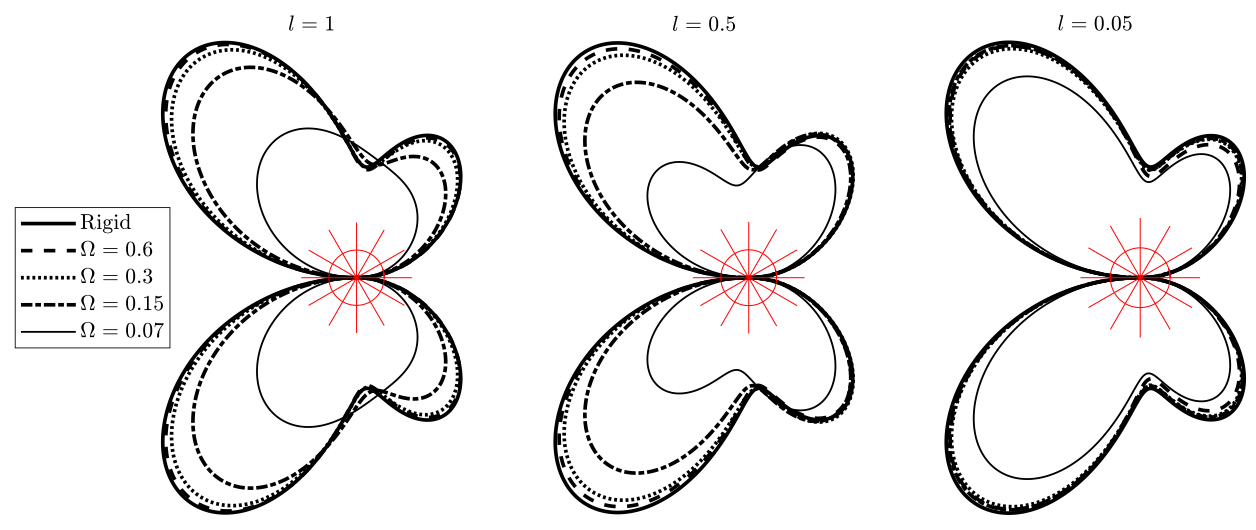

(a)
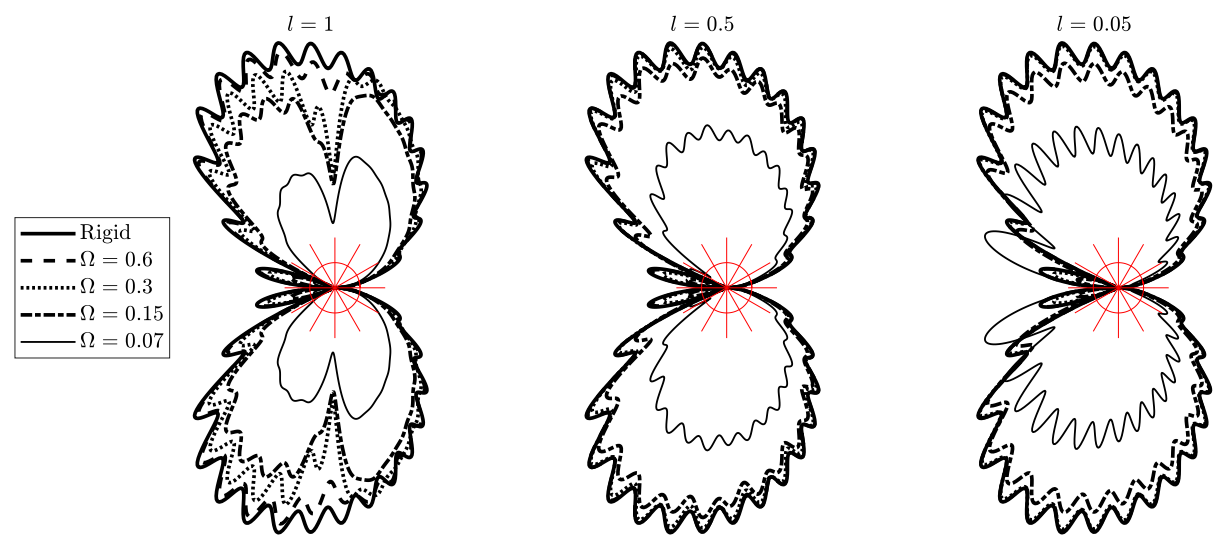

(b)

Figure 13: (a) Far-field directivity for $k_{0}=5, \epsilon=0.0021$ and different $l$, the red lines show polar angles in increments of $30^{\circ}$. (b) Same but for $k_{0}=50$. In both cases, the far-field is due to a quadrupole located at $x=1, y=0.02$ above a plate. 
a linear system for the unknown coefficients of the vibrational modes, and which can be given explicitly in terms of special functions (these have builtin routines in most numerical software packages). The method is simple, fast and flexible, allowing a mixture of elastic plates with different physical parameters, as well as rigid plates, to be treated collectively in the same manner. Though we have focused on collinear plates, the method can easily be adapted to more complicated geometries (see for example [5] for the rigid case or [4] for curved boundaries), a topic which will be explored in future work. The method can be applied to situations where an approximate Wiener-Hopf solution is very difficult to obtain, such as matrix problems arising from multiple points at which the type of boundary condition changes or due to the complexity of the elastic kernel function. When compared with typical boundary element methods, this new spectral approach has the clear advantage of avoiding the evaluation of singular integrals and is more flexible than previous BEM methods [3], which have treated the case of a single elastic plate. The method has been applied to semi-infinite plates [5], but not in the case of elastic boundary conditions (which has been well studied analytically [16]). Whilst semi-infinite stationary plates are useful approximations in aeroacoustic scattering problems, they are however not as convenient for elastic plates since a semi-infinite elastic plate cannot appropriately replicate structural resonances which will occur in practice, and thus we have not considered them here. The approach adopted here is unlikely to work in the semi-infinite elastic case since the spectrum of the biharmonic operator on a semi-infinite strip is no longer discrete, meaning we can no longer use the eigenfunctions as a suitable basis. Nevertheless, future work may aim to tackle this case directly using Eq. (2) and Eq. (3).

It should be mentioned that the method converges (at least) exponentially for rigid plates, however, this is not the case for elastic plates. This is due to the approximation properties of the chosen basis (the smooth vibrational modes), which were chosen to cope with the boundary conditions given by Eq. (2) and Eq. (3) involving a fourth order derivative and cannot capture the endpoint singularities. Nevertheless, the method still converges algebraically and can easily gain a few digits of precision for modest $N$. Future work will seek to explore different choices of basis functions, which we anticipate may increase the rate of convergence at the expense of a more demanding global relation. Future work will also consider the extension of this method to three dimensions, which has been successfully achieved by the previous BEM approach for a single structure [21]. 
We have illustrated this new method by considering the acoustic fields generated by collinear elastic plates subjected to incident plane waves or turbulent near-field sources (other types of sources or scattering problems can also be handled). A feature clearly evident from the illustrated results for partially elastic plates, that is plates with a rigid fore section and elastic aft section, is that the effectiveness of elasticity on reducing aerodynamic trailing-edge noise can be significantly altered at mid and high frequencies even when only a small section of the overall plate at the trailing edge is elastic. By only altering a partial section of the plate to be elastic, this has the potential therefore to significantly reduce noise but also to allow greater aerodynamic control than if the plates were fully elastic (since fully elastic plates have highly complex behaviour in flow [12, 24]). Of course, further work would be required to understand the full impact of partially elastic trailing edges on the aerodynamics and to balance with acoustic reductions.

\section{Acknowledgements}

This work was supported by EPSRC grant EP/L016516/1 (M.J.C) and EPSRC Early-Career Fellowship EP/P015980/1 (L.J.A.). Example code will also be made available on M.J.C.'s website.

[1] L.J. Ayton. Acoustic scattering by a finite rigid plate with a poroelastic extension. Journal of Fluid Mechanics, 791:414-438, 2016.

[2] J.P. Boyd. Chebyshev and Fourier spectral methods. Courier Corporation, 2001.

[3] A.V.G. Cavalieri, W.R. Wolf, and J.W. Jaworski. Numerical solution of acoustic scattering by finite perforated elastic plates. Proceedings of the Royal Society of London A: Mathematical, Physical and Engineering Sciences, 472, 2016.

[4] M. J. Colbrook. Extending the unified transform: curvilinear polygons and variable coefficient PDEs. IMA Journal of Numerical Analysis, 2018.

[5] M. J. Colbrook, L. J. Ayton, and A. S. Fokas. The unified transform for mixed boundary condition problems in unbounded domains. Proceedings of the Royal Society of London A: Mathematical, Physical and Engineering Sciences, 475, 2019. 
[6] M.J. Colbrook, N. Flyer, and B. Fornberg. On the Fokas method for the solution of elliptic problems in both convex and non-convex polygonal domains. Journal of Computational Physics, 374:996-1016, 2018.

[7] M.J. Colbrook, T.S. Fokas, and P. Hashemzadeh. A Hybrid AnalyticalNumerical Technique for Elliptic PDEs. SIAM Journal on Scientific Computing, 41(2):A1066-A1090, 2019.

[8] D.G. Crighton and F.G. Leppington. Scattering of aerodynamic noise by a semi-infinite compliant plate. Journal of Fluid Mechanics, 43:721-736, 1970.

[9] D.G. Crowdy. Fourier-Mellin transforms for circular domains. Computational Methods and Function Theory, 15(4):655-687, 2015.

[10] D.G. Crowdy. A transform method for Laplace's equation in multiply connected circular domains. IMA Journal of Applied Mathematics, 80(6):1902-1931, 2015.

[11] A.S. Fokas. A unified approach to boundary value problems. SIAM, 2008.

[12] R.E. Gordnier. High fidelity computational simulation of a membrane wing airfoil. Journal of Fluids and Structures, 25:897-917, 2009.

[13] P. Hashemzadeh, A.S. Fokas, and S.A. Smitheman. A numerical technique for linear elliptic partial differential equations in polygonal domains. Proc. R. Soc. A, 471(2175):20140747, 2015.

[14] M.S. Howe. Structural and acoustic noise produced by turbulent flow over an elastic trailing edge. Proceedings of the Royal Society of London A: Mathematical, Physical and Engineering Sciences, 442:533-554, 1993.

[15] M.S. Howe. Acoustics of fluid-structure interactions. Cambridge University Press, 1998.

[16] J.W. Jaworski and N. Peake. Aerodynamic noise from a poroelastic edge with implications for the silent flight of owls. Journal of Fluid Mechanics, 723:456-479, 2013.

[17] E. Luca and D.G. Crowdy. A transform method for the biharmonic equation in multiply connected circular domains. IMA Journal of Applied Mathematics, 2018. 
[18] S. Moreau and M. Roger. Backscattering correction and further extensions of Amiet's trailing-edge noise model. part ii: Application. Journal of Sound and Vibration, 323:397-425, 2009.

[19] B. Noble. Methods based on the Wiener-Hopf technique for the solution of partial differential equations. Chelsea Pub Co, 1988.

[20] F.W.J. Olver, D.W. Lozier, R.F. Boisvert, and C.W. Clark. NIST handbook of mathematical functions. Cambridge University Press, 2010.

[21] C. Pimenta, W.R. Wolf, and A.V.G. Cavalieri. A fast numerical framework to compute acoustic scattering by poroelastic plates of arbitrary geometry. Journal of Computational Physics, 373:763-783, 2018.

[22] K.F. Riley, M.P. Hobson, and S.J. Bence. Mathematical methods for physics and engineering: a comprehensive guide. Cambridge university press, 2006.

[23] J.F.M. Scott. Acoustic scattering by a finite elastic strip. Philosophical Transactions of the Royal Society of London A: Mathematical, Physical and Engineering Sciences, 338:145-167, 1992.

[24] A. Song, X. Tian, E. Israeli, R. Galvao, K. Bishop, S. Swartz, and K. Breuer. Aeromechanics of membrane wings with implications for animal flight. AIAA Journal, 46:2096-2106, 2008.

[25] E.A. Spence. Boundary value problems for linear elliptic PDEs. PhD thesis, University of Cambridge, 2011.

[26] I.V. Sturova and L.A. Tkacheva. The motion of pressure distribution over a free surface near the edge of ice sheet. Earth and Environmental Science, 193:012065, 2018.

[27] L.A. Tkacheva. Scattering of surface waves by the edge of a floating elastic plate. Journal of Applied Mechanics and Technical Physics, 42:638646, 2001.

[28] L.N. Trefethen. Spectral methods in MATLAB. Siam, 2000.

[29] C. Zhao, C. Hu, Y. Wei, J. Zhang, and W. Huang. Diffraction of surface waves by floating elastic plates. Journal of Fluids and Structures, 24:231-249, 2008. 